\title{
Tunnelling magnetoresistance of the half-metallic compensated ferrimagnet $\mathrm{Mn}_{\mathbf{2}} \mathbf{R u} \mathrm{u}_{\mathbf{x}} \mathrm{Ga}$
}

K. Borisov, D. Betto, Y.-C. Lau, C. Fowley, A. Titova, N. Thiyagarajah, G. Atcheson, J. Lindner, A. M. Deac, J. M.

D. Coey, P. Stamenov, and K. Rode

Citation: Appl. Phys. Lett. 108, 192407 (2016);

View online: https://doi.org/10.1063/1.4948934

View Table of Contents: http://aip.scitation.org/toc/apl/108/19

Published by the American Institute of Physics

\section{Articles you may be interested in}

The zero-moment half metal: How could it change spin electronics?

AIP Advances 6, 055601 (2016); 10.1063/1.4943756

Narrow-band tunable terahertz emission from ferrimagnetic $\mathrm{Mn}_{3-\mathrm{x}} \mathrm{Ga}$ thin films

Applied Physics Letters 109, 032403 (2016); 10.1063/1.4958855

Giant spontaneous Hall effect in zero-moment $\mathrm{Mn}_{2} \mathrm{Ru}_{\mathrm{x}} \mathrm{Ga}$

Applied Physics Letters 106, 122402 (2015); 10.1063/1.4913687

Basics and prospective of magnetic Heusler compounds

APL Materials 3, 041518 (2015); 10.1063/1.4917387

$\mathrm{Mn}_{3} \mathrm{Ga}$, a compensated ferrimagnet with high Curie temperature and low magnetic moment for spin torque transfer applications

Applied Physics Letters 90, 152504 (2007); 10.1063/1.2722206

Magnetic and electronic properties of $\mathrm{D}_{22}-\mathrm{Mn}_{3} \mathrm{Ge}(001)$ films

Applied Physics Letters 101, 132410 (2012); 10.1063/1.4754123

\section{Scilight}

Sharp, quick summaries illuminating the latest physics research

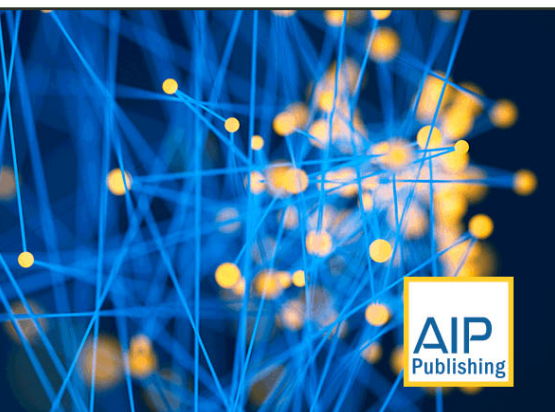




\title{
Tunnelling magnetoresistance of the half-metallic compensated ferrimagnet $\mathrm{Mn}_{\mathbf{2}} \mathrm{Ru}_{\mathrm{x}} \mathrm{Ga}$
}

\author{
K. Borisov, ${ }^{1, a)}$ D. Betto, ${ }^{1, b)}$ Y.-C. Lau, ${ }^{1, c)}$ C. Fowley, ${ }^{2}$ A. Titova, ${ }^{2}$ N. Thiyagarajah,,${ }^{1, d)}$ \\ G. Atcheson, ${ }^{1}$ J. Lindner, ${ }^{2}$ A. M. Deac, ${ }^{2}$ J. M. D. Coey, ${ }^{1}$ P. Stamenov, ${ }^{1}$ and K. Rode ${ }^{1}$ \\ ${ }^{1}$ School of Physics, AMBER and CRANN, Trinity College, Dublin 2, Ireland \\ ${ }^{2}$ Helmholtz-Zentrum Dresden-Rossendorf, Institute of Ion Beam Physics and Materials Research, Dresden, \\ Germany
}

(Received 31 March 2016; accepted 27 April 2016; published online 10 May 2016)

\begin{abstract}
Tunnel magnetoresistance ratios of up to $40 \%$ are measured between $10 \mathrm{~K}$ and $300 \mathrm{~K}$ when the highly spin-polarized compensated ferrimagnet, $\mathrm{Mn}_{2} \mathrm{Ru}_{x} \mathrm{Ga}$, is integrated into $\mathrm{MgO}$-based perpendicular magnetic tunnel junctions. Temperature and bias dependences of the tunnel magnetoresistance effect, with a sign change near $-0.2 \mathrm{~V}$, reflect the structure of the $\mathrm{Mn}_{2} \mathrm{Ru}_{x} \mathrm{Ga}$ interface density of states. Despite magnetic moment vanishing at a compensation temperature of $200 \mathrm{~K}$ for $x \approx 0.8$, the tunnel magnetoresistance ratio remains non-zero throughout the compensation region, demonstrating that the spin-transport is governed by one of the Mn sub-lattices only. Broad temperature range magnetic field immunity of at least $0.5 \mathrm{~T}$ is demonstrated in the same sample. The high spin polarization and perpendicular magnetic anisotropy make $\mathrm{Mn}_{2} \mathrm{Ru}_{x} \mathrm{Ga}$ suitable for applications in both non-volatile magnetic random access memory cells and terahertz spin-transfer oscillators. Published by AIP Publishing.

[http://dx.doi.org/10.1063/1.4948934]
\end{abstract}

Spin-torque nano-oscillators (STNO) based on transitionmetal ferromagnets (FMs) currently offer a route for lowinput power, ${ }^{1}$ high-output power, ${ }^{2}$ and nano-scale wireless transceivers with frequency tunability up to $65 \mathrm{GHz},{ }^{3}$ compatible with current CMOS technology. ${ }^{4}$ The upper frequency limit for devices based on transition metal ferromagnets is limited by unchangeable material properties. For future applications, transmission frequencies in the terahertz gap are required. For this, one can exploit high anisotropy Heusler compounds ${ }^{5}$ which display magnetic resonance frequencies in the range of 100's of GHz. ${ }^{6,7}$ The key material properties for integration in STNO geometries, where magnetisation is manipulated by spin-polarized currents, are a high interface transport spin polarisation $P$; low saturation magnetization $M_{\mathrm{s}}$; low Gilbert damping $\alpha$; and a high effective magnetic field. ${ }^{8}$ Antiferromagnets (AFMs) fulfill many of these requirements, and significant progress in the electrical switching of AFMs has been made recently, ${ }^{9}$ but the effect is currently limited by the magnitude of the anisotropic magnetoresistance. Here, we pursue a different approach by incorporating in magnetic tunnel junctions (MTJs), a material which behaves magnetically like an AFM, but in terms of transport acts like a ferromagnet (FM). This is the compensated half-metallic ferrimagnet (CHFM) - a class of materials predicted in 1995 by van Leuken and de Groot, ${ }^{10,11}$ for which first experimental evidence was only reported in 2014 (Ref. 12) for a near-cubic Heusler alloy of Mn, Ga, and Ru (MRG). The compound exhibits the ideal combination of negligible $M_{\mathrm{s}}$, high spin

\footnotetext{
a)borisovk@tcd.ie

${ }^{b}$ Present address: European Synchrotron Radiation Facility (ESRF), BP 220, F-38043 Grenoble Cedex, France

${ }^{c}$ Present address: National Institute for Materials Science, Tsukuba 3050047, Japan

${ }^{d)}$ Present address: Globalfoundries, Singapore, 60 Woodlands Industrial Park D Street 2, Singapore 738406
}

polarization, and a high magnetic ordering temperature $T_{\mathrm{c}}$, indicative of a strong exchange-field $H_{\mathrm{ex}}{ }^{13}$ In compensated ferrimagnets, the exchange mode resonance frequency (EMR) is written ${ }^{14} f=\left(\mu_{0} \gamma / 2 \pi\right) \sqrt{H_{\mathrm{a}}\left(H_{\mathrm{a}}+2 H_{\mathrm{ex}}\right)} \approx\left(\mu_{0} \gamma / 2 \pi\right) \sqrt{2 H_{\mathrm{a}} H_{\mathrm{ex}}}$ (for $H_{\mathrm{ex}} \gg H_{\mathrm{a}}$ ), where $H_{\mathrm{a}}$ is the sublattice anisotropy field. The EMR frequency of MRG is of order of $0.7 \mathrm{THz},{ }^{15}$ inferred from the sublattice magnetization, the Curie temperature, and the anisotropy constant $K_{1_{\text {eff }}} \cdot 16$

In this letter, we demonstrate that MRG can be incorporated in perpendicular anisotropy (PMA) MTJ stacks which exhibit sufficient tunneling magnetoresistance (TMR) ratios to observe current induced magnetisation dynamics in future devices, and hence constitutes a cornerstone of future spin electronic devices. Furthermore, we show that the TMR is insensitive to the compensation of the ferrimagnetic electrode.

MRG-based MTJs were deposited using a "Shamrock" fully automated sputter deposition tool, with a base pressure of $1 \times 10^{-8}$ mbar. MRG was grown by co-sputtering from a $\mathrm{Mn}_{2} \mathrm{Ga}$ and a Ru target. The growth temperature for the MRG electrode was in the range from 300 to $350^{\circ} \mathrm{C}$, while the rest of the stack was deposited at room temperature. Further details of the growth of MRG can be found elsewhere. ${ }^{12,15}$ Following the deposition of $\sim 40 \mathrm{~nm}$ of MRG, a dusting layer of varying thickness of $\mathrm{Al}$ was grown, before depositing the $\mathrm{MgO}$ insulating tunnel barrier, and finally a composite top electrode constituted of $\mathrm{CoFeB}(1.0) / \mathrm{Ta}(0.3) / \mathrm{CoFeB}(0.9) / \mathrm{MgO}(0.7) / \mathrm{Ta}(3.0) /$ $\mathrm{Ru}(4.0)$, (thicknesses in $\mathrm{nm}$ ), as illustrated in Fig. 1. The composite top electrode was chosen as it provides an $\mathrm{MgO} / \mathrm{CoFeB}$ interface with PMA that is more stable against surface roughness and ensures better thermal stability. ${ }^{17}$ The $\mathrm{Al}$ dusting layer acts as a diffusion barrier for the Mn atoms.

The samples were patterned into $6 \times 6 \mu \mathrm{m}^{2}$ and $20 \times 20 \mu \mathrm{m}^{2}$ junctions, using standard UV lithography techniques. $\mathrm{A} \mathrm{Cr} / \mathrm{Au}$ top contact for electrical connections was defined by electron- 

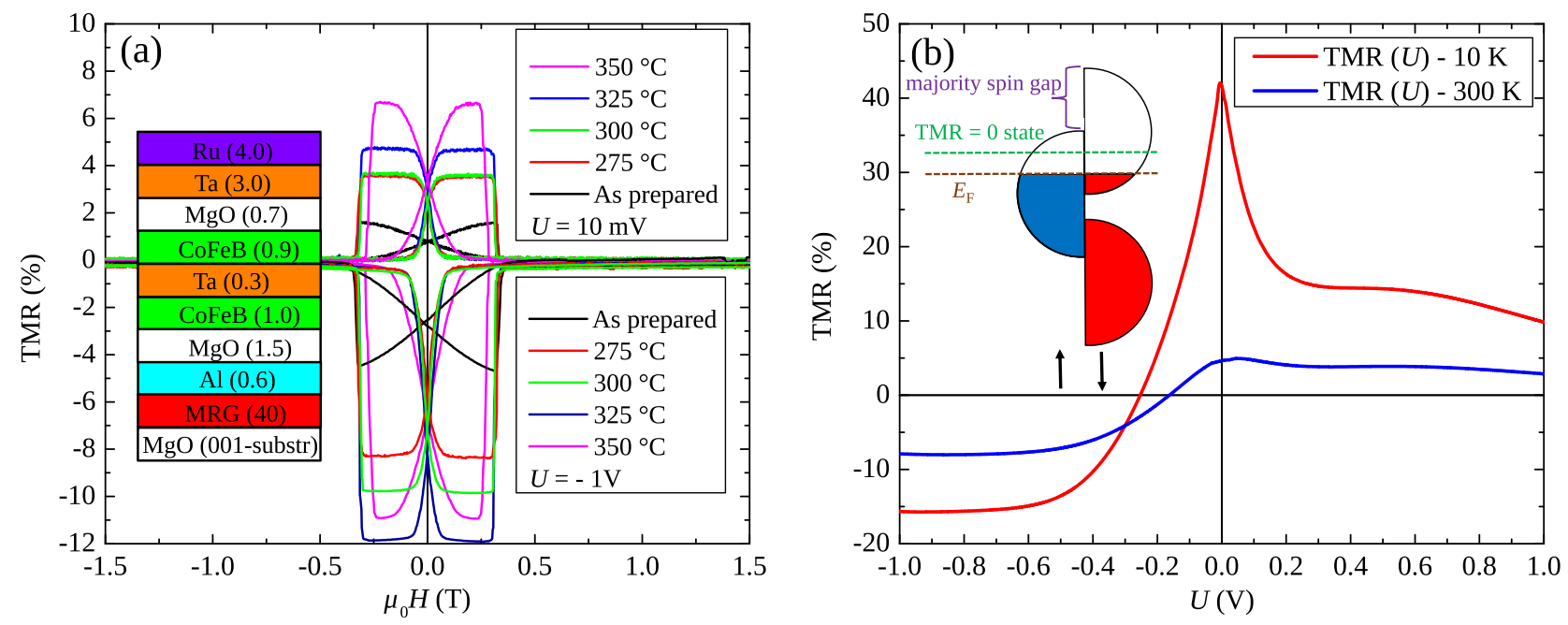

FIG. 1. (a) Room temperature $(300 \mathrm{~K}) \mathrm{TMR}$ data of the $x=1.0$ sample annealed at different temperatures. Positive TMR is observed at applied bias $U=10 \mathrm{mV}$, and negative at $U=-1 \mathrm{~V}$. (b) TMR $(U)$ scans at $300 \mathrm{~K}$ and $10 \mathrm{~K}$ for a chip annealed at $350^{\circ} \mathrm{C}$. The top left inset represents the spin split density of states of MRG for $x=1.0 .^{12}$

beam deposition and lift-off. We also fabricated MTJs without the Al dusting layer. After patterning, the samples were annealed for $1 \mathrm{~h}$ in a magnetic annealing furnace $\left(\mu_{0} \vec{H}\right.$ $=0.8 \mathrm{~T} \|[001]_{\mathrm{MgO}}$ ), in temperatures ranging from 275 to $350^{\circ} \mathrm{C}$. All magnetoelectrical measurements were carried out with a Keithley 2400 source-meter, with the sample in a Quantum Design Physical Properties Measurement System. The temperature was varied from 10 to $400 \mathrm{~K}$, and the applied magnetic field from 0 to $14 \mathrm{~T}$.

First, we analyze the effect of the annealing temperature, $T_{\mathrm{a}}$, on the magnetic state and TMR ratios for a sample with $x=1.0$ (panel (a) on Fig. 1). As-deposited, the CoFe composite top electrode does not exhibit strong PMA, as can be seen from the linear rotation of its magnetization between $\pm 0.3 \mathrm{~T}$. As $T_{\mathrm{a}}$ is increased, the top electrode crystallizes and the PMA increases, evidenced by the sharper transition of the free layer around zero field. When $T_{\mathrm{a}}$ exceeds $325^{\circ} \mathrm{C}$, the PMA of the top $\mathrm{CoFe}$ electrode degrades, resulting in a poorly defined antiparallel magnetic state. The annealing-induced change of the magnetic state is accompanied by an increase of the TMR ratio. At low bias $(U=10 \mathrm{mV})$, the as-grown sample exhibits $\approx 1.7 \%$ TMR at room-temperature (RT), increasing to $\approx 6.7 \%$ after annealing at $350{ }^{\circ} \mathrm{C}$. At high negative bias $(U=-1 \mathrm{~V})$, the variation is from -4 to $-12 \%$, for the as-grown sample and the sample with $T_{\mathrm{a}}=325^{\circ} \mathrm{C}$, respectively. In addition to inducing PMA of the top electrode, the thermal treatment drives MRG towards its thermodynamic equilibrium. The most stable phase in the cubic $\mathrm{Mn}-\mathrm{Ga}-\mathrm{Ru}$ phase-diagram favors Mn-Ga anti-sites, with an associated increase of the spin polarization. ${ }^{18}$ Unfortunately, the MRG/Al interface does not possess the required symmetry so that $\Delta_{1}$ spin filtering through the $\mathrm{MgO}$ barrier is not efficient in our stack structure.

Panel (b) on Fig. 1 shows the TMR ratio at $10 \mathrm{~K}$ and $300 \mathrm{~K}$ as a function of applied bias $U$. Positive (negative) bias corresponds to electrons tunneling from (into) MRG. Around $U=0$, a pronounced zero-bias anomaly is observed, resulting in a maximum TMR of $40 \%$. For $U>0$, the TMR ratio decreases with a line-shape similar, albeit steeper, to conventional MTJs, with a local plateau around $U \approx 0.55 \mathrm{~V}$ which is not usually observed in standard MTJ devices. ${ }^{19}$ The bias dependence for $U<0$ is more striking. The TMR ratio decreases rapidly with increasing negative bias and reaches zero for $U=-240 \mathrm{mV}$. It then continues to decrease and reaches $-16 \%$ for $U=-1 \mathrm{~V}$. We interpret this as follows: At low bias, electrons are tunneling between the Fermi levels of MRG and $\mathrm{CoFe}$, and the normal zero-bias behavior is observed. As $U$ decreases, the tunneling final state shifts from the MRG spin $\uparrow$ pocket (attributed to the Mn $4 c$ site) to the spin $\downarrow$ pocket (attributed to the Mn $4 a$ site). The TMR $(-1 \mathrm{~V})$ is more temperature robust because electrons tunnel to the MRG majority spin gap. The TMR-voltage dependence is consistent with the simplified representation of the MRG density of states. ${ }^{12}$ The same qualitative behavior was observed on a sample with no insertion layer; hence, contributions from quantum well tunneling states ${ }^{20,21}$ and surface density of states modification ${ }^{22}$ by the $\mathrm{Al}$ insertion layer can be ruled out. This sample demonstrated an order of magnitude smaller TMR ratio due to the oxidation of the MRG interface ( $\mathrm{TMR} \approx 0.5 \%$ at $\mathrm{RT}$ ), highlighting the importance of the insertion layer. We found that a $0.3 \mathrm{~nm} \mathrm{Al}$ insertion layer was ineffective, but that $0.6 \mathrm{~nm}$ was sufficient to greatly increase the TMR. Despite the Al diffusion barrier, a small concentration of Mn impurities was detected in the $\mathrm{MgO}$ by time-of-flight secondary ion-mass spectroscopy. ${ }^{18}$ The TMR $(U)$ at $300 \mathrm{~K}$ shows similar behavior. However, the low bias TMR is significantly reduced, while the high negative bias TMR $(U=-1 \mathrm{~V})$ exhibits good temperature stability.

In Fig. 2, we show the evolution of the TMR of a sample annealed at $325^{\circ} \mathrm{C}$ with $T$ and $U$. There are two main conclusions to be drawn from the TMR behavior. First, the lowbias TMR decreases rapidly with temperature, indicating that the tunneling process is predominantly incoherent. In agreement with this picture, the parallel-state resistance drops by $30 \%$ between 10 and $300 \mathrm{~K}$. The spin-independent conductance of our MTJs could be analyzed following the multi-step tunneling model by Shang, ${ }^{23,24}$ but cannot explain the strong zero-bias TMR decrease, since the higher average 


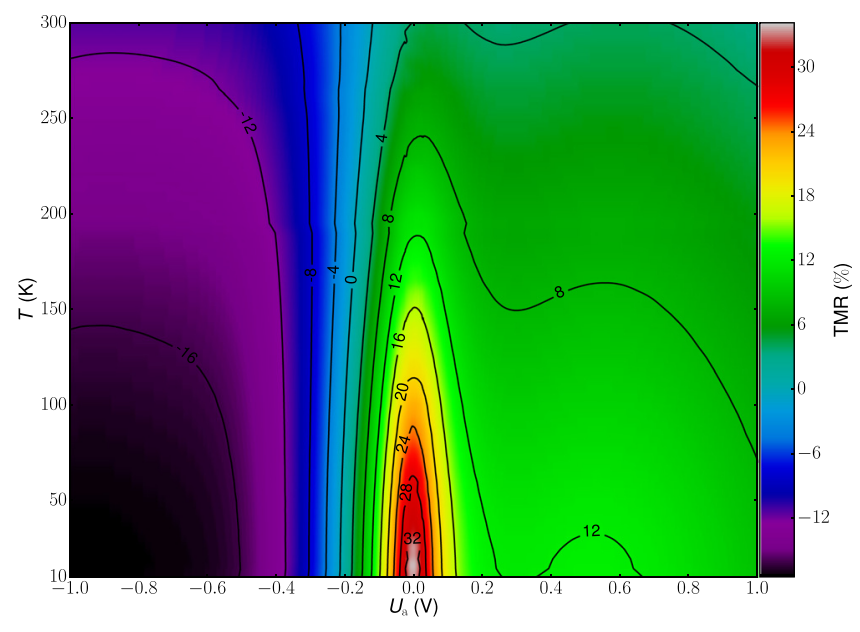

FIG. 2. TMR as a function of temperature and bias. The MRG composition is $x=1$ and the Al dusting layer thickness is $0.6 \mathrm{~nm}$. The junction was annealed at $325^{\circ} \mathrm{C}$.

number of tunneling steps found at $U=-1 \mathrm{~V}$ does not correlate with the significantly more temperature-robust TMR $[N(10 \mathrm{mV}) \approx 2.2 ; N(-1 \mathrm{~V}) \approx 2.8]$. Tunneling in our MTJs cannot be described in the simple, incoherent picture. Second, there is a clear dip in the TMR around $U \approx+0.3 \mathrm{~V}$. This indicates that in addition to the incoherent process, there is a resonant contribution via impurities in the barrier, or at its interfaces. ${ }^{25,26}$ The resonant contribution to the tunneling process is a possible reason why thermal annealing affects the TMR ratio differently at $U=10 \mathrm{mV}$ and $U=$ $-1 \mathrm{~V}$ (see Fig. 1(a)).

Finally, we demonstrate that TMR can be achieved with an electrode which exhibits strictly zero net magnetic moment. We start with a discussion on the behavior of the MRG magnetic moment $M_{\mathrm{MRG}}$ and its spin polarization $P_{\text {MRG }}$ during a thermal scan through compensation $\left(T_{\text {comp }}\right)$. The relative orientation of $M_{\mathrm{MRG}}$ and $P_{\mathrm{MRG}}$ always changes upon crossing $T_{\text {comp }}$. However, the direction of $M_{\mathrm{MRG}}$ depends critically on the magnitude of the applied field $\left(\mu_{0} H\right)$ with respect to the MRG coercivity $\left(\mu_{0} H_{\mathrm{c}}^{\mathrm{MRG}}\right)$. First, when $\left|\mu_{0} H\right|>\mu_{0} H_{\mathrm{c}}^{\mathrm{MRG}}, M_{\mathrm{MRG}}$ follows $\mu_{0} H$, as does the magnetically soft $\mathrm{CoFe}$ electrode. Consequently, $P_{\mathrm{MRG}}$ flips with respect to $P_{\mathrm{CoFe}}$ and the TMR changes sign. ${ }^{27}$ The decrease of the TMR near $T_{\text {comp }}$ (Fig. 3(a): $200 \mathrm{~K}$ ) is due to inevitable lateral variations of composition and strain. Second, when $\left|\mu_{0} H\right|<\mu_{0} H_{\mathrm{c}}^{\mathrm{MRG}}, M_{\mathrm{MRG}}$ changes sign with respect to $\mu_{0} H$. Therefore, the direction of $P_{\mathrm{MRG}}$ and $P_{\mathrm{CoFe}}$ remain parallel and the TMR sign does not change.

In Fig. 3(a), we show full TMR loops below (165 K), near $(200 \mathrm{~K})$, and above $(235 \mathrm{~K}) T_{\text {comp }}$. The sign of the TMR is changed when crossing $T_{\text {comp }}$, demonstrating the reversal of $P_{\mathrm{MRG}}$ with respect to $P_{\mathrm{CoFe}}$, i.e., $P_{\mathrm{MRG}}$ is parallel to $P_{\mathrm{CoFe}}$ below $T_{\text {comp }}$ and antiparallel above.

In order to investigate further the behavior at $T_{\text {comp }}$, we conducted temperature scans through $T_{\text {comp }}$ with $\left|\mu_{0} H\right| \ll \mu_{0} H_{\mathrm{c}}^{\mathrm{MRG}}$. Fig. 3(b) shows minor loops at different temperatures above, near, and below $T_{\text {comp }}$, after saturating the sample well above $T_{\text {comp }}$. The maximum applied field is only $\mu_{0} H= \pm 0.5 \mathrm{~T}$. The TMR remains almost constant through $T_{\text {comp }}$ and there is no sign change.

We now turn to the TMR when $M_{\mathrm{MRG}}=0$ (inset of Fig. 3(b)). After having saturated the two electrodes in $\mu_{0} H=14 \mathrm{~T}$ at $T=10 \mathrm{~K}\left(P_{\mathrm{MRG}}\right.$ parallel $\left.P_{\mathrm{CoFe}}\right)$, we recorded the MTJ resistance in $\mu_{0} H=-0.5 \mathrm{~T}$, corresponding to the antiparallel alignment of the two electrodes, while scanning the temperature from 10 to $300 \mathrm{~K}$. We then performed the same temperature scan with parallel alignment to obtain the TMR ratio as a function of temperature (black curve). The experiment was then repeated, this time saturating at $T=300 \mathrm{~K}\left(P_{\mathrm{MRG}}\right.$ antiparallel $\left.P_{\mathrm{CoFe}}\right)$ and measuring from 300 to $10 \mathrm{~K}$. Other than the overall temperature dependence of the TMR, there is no sign change. This measurement proves that the TMR is finite, and unchanged, even when the net magnetization of MRG is strictly zero. This is a strong evidence that only one of the two magnetic sublattices contributes to the magnetotransport close to the MRG Fermi level. It also highlights a fundamental difference between an antiferromagnet and a compensated half-metallic ferrimagnet.

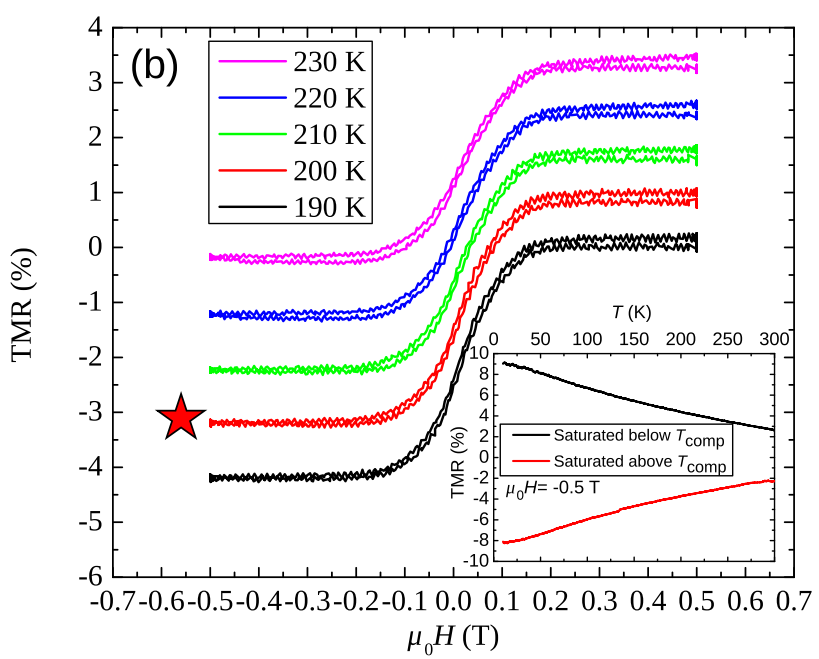

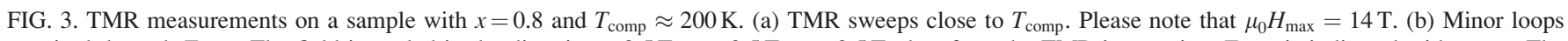

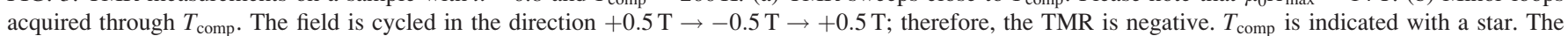

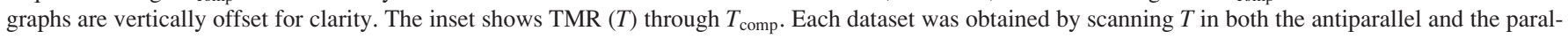
lel configuration, after saturation at $T=10 \mathrm{~K}$ (black line) and $T=300 \mathrm{~K}$ (red line). 
This demonstration of a TMR effect using a truly compensated ferrimagnet opens both technological opportunities as well as further possibilities in fundamental experimental spin electronics. The nature of spin injection, and transfer of angular momentum, in a CHFM is currently poorly understood, mainly due to the lack of a good experimental testing ground. We have shown that the compensated ferrimagnet MRG, which demonstrates clear signs of transport half metallicity, provides sufficiently high TMR ratios to be considered a candidate for future STNO. At the compensation point MRG displays a large external field immunity, at least $0.5 \mathrm{~T}$ which can be exploited for magnetic memory elements. The observed TMR is much lower than might be expected for a half metal. We attribute this to barrier imperfections, specifically residual Mn diffusion, which will reduce the tunneling spin polarization and increase the temperature dependence of the TMR. Further investigation by spin-resolved photoemission spectroscopy is needed in order to clarify what is the temperature decrease of the MRG/barrier interface spin polarization. The observation of large TMR values is stimulating in the fact that it would provide a way to electrically detect resonances of both the EMR- and the FMR-like modes. The next step is therefore to reduce the barrier thickness and fabricate sub- $\mu \mathrm{m}$ devices allowing for the detection of spin-transfer induced dynamics.

K.B. and P.S. acknowledge financial support from Science Foundation Ireland (SFI) within SSPP (11/SIRG/I2130). K.B., D.B., Y.C.L., J.M.D.C., P.S., and K.R. were supported by SFI through AMBER, and from Grant No. 13/ERC/I2561. D.B. acknowledges support from IRCSET. A.T. acknowledges support from the Helmholtz-Zentrum Dresden-Rossendorf Summer student program. C.F. and A.M.D. acknowledge support from the Helmholtz Young Investigator Initiative Grant No. VH-N6-1048. Support by the Nanofabrication Facilities Rossendorf at Ion Beam Center is gratefully acknowledged.

${ }^{1}$ Z. Zeng, G. Finocchio, B. Zhang, P. K. Amiri, J. A. Katine, I. N. Krivorotov, Y. Huai, J. Langer, B. Azzerboni et al., Sci. Rep. 3, 1426 (2013).

${ }^{2}$ H. Maehara, H. Kubota, Y. Suzuki, T. Seki, K. Nishimura, Y. Nagamine, K. Tsunekawa, A. Fukushima, H. Arai et al., Appl. Phys. Express 7(2), 023003 (2014).
${ }^{3}$ S. Bonetti, P. Muduli, F. Mancoff, and J. Åkerman, Appl. Phys. Lett. 94(10), 102507 (2009).

${ }^{4}$ S. Tamaru, H. Kubota, K. Yakushiji, S. Yuasa, and A. Fukushima, Sci. Rep. 5, 18134 (2015).

${ }^{5}$ T. Graf, C. Felser, and S. S. Parkin, Prog. Solid State Chem. 39(1), 1 (2011).

${ }^{6}$ S. Mizukami, F. Wu, A. Sakuma, J. Walowski, D. Watanabe, T. Kubota, X. Zhang, H. Naganuma, M. Oogane et al., Phys. Rev. Lett. 106(11), 117201 (2011).

${ }^{7}$ C. Fowley, S. Ouardi, T. Kubota, O. Yildirim, A. Neudert, K. Lenz, V. Sluka, J. Lindner, J. M. Law et al., J. Phys. D: Appl. Phys. 48(16), 164006 (2015).

${ }^{8}$ M. Oogane, T. Kubota, Y. Kota, S. Mizukami, H. Naganuma, A. Sakuma, and Y. Ando, Appl. Phys. Lett. 96(25), 252501 (2010).

${ }^{9}$ P. Wadley, B. Howells, J. Železný, C. Andrews, V. Hills, R. P. Campion, V. Novák, K. Olejník, F. Maccherozzi et al., Science 351(6273), 587 (2016).

${ }^{10}$ H. van Leuken and R. A. de Groot, Phys. Rev. Lett. 74, 1171 (1995).

${ }^{11}$ S. Wurmehl, H. C. Kandpal, G. H. Fecher, and C. Felser, J. Phys.: Condens. Matter 18(27), 6171 (2006).

${ }^{12}$ H. Kurt, K. Rode, P. Stamenov, M. Venkatesan, Y.-C. Lau, E. Fonda, and J. M. D. Coey, Phys. Rev. Lett. 112, 027201 (2014).

${ }^{13}$ D. Betto, K. Rode, N. Thiyagarajah, Y.-C. Lau, K. Borisov, G. Atcheson, M. Žic, T. Archer, P. Stamenov et al., AIP Adv. 6(5), 055601 (2016).

${ }^{14}$ J. Kaplan and C. Kittel, J. Chem. Phys. 21(4), 760 (1953).

${ }^{15}$ N. Thiyagarajah, Y.-C. Lau, D. Betto, K. Borisov, J. M. D. Coey, P. Stamenov, and K. Rode, Appl. Phys. Lett. 106(12), 122402 (2015).

${ }^{16}$ D. Betto, N. Thiyagarajah, Y.-C. Lau, C. Piamonteze, M.-A. Arrio, P. Stamenov, J. M. D. Coey, and K. Rode, Phys. Rev. B 91, 094410 (2015).

${ }^{17}$ H. Sato, M. Yamanouchi, S. Ikeda, S. Fukami, F. Matsukura, and H. Ohno, Appl. Phys. Lett. 101(2), 022414 (2012).

${ }^{18}$ M. Žic, K. Rode, N. Thiyagarajah, Y.-C. Lau, D. Betto, J. M. D. Coey, S. Sanvito, K. J. O'Shea, C. A. Ferguson et al., Phys. Rev. B 93, 140202 (2016).

${ }^{19}$ S. Yuasa, T. Nagahama, A. Fukushima, Y. Suzuki, and K. Ando, Nat. Mater. 3(12), 868 (2004).

${ }^{20}$ A. Vedyayev, N. Ryzhanova, C. Lacroix, L. Giacomoni, and B. Dieny, Europhys. Lett. 39(2), 219 (1997).

${ }^{21}$ J. S. Moodera, J. Nowak, L. R. Kinder, P. M. Tedrow, R. J. M. van de Veerdonk, B. A. Smits, M. van Kampen, H. J. M. Swagten, and W. J. M. de Jonge, Phys. Rev. Lett. 83, 3029 (1999).

${ }^{22}$ J. M. De Teresa, A. Barthlmy, A. Fert, J. P. Contour, F. Montaigne, and P. Seneor, Science 286(5439), 507 (1999).

${ }^{23}$ C. H. Shang, J. Nowak, R. Jansen, and J. S. Moodera, Phys. Rev. B 58, R2917 (1998).

${ }^{24}$ L. Glazman and K. Matveev, Z. Eksp. Teor. Fiz. 94, 343 (1988).

${ }^{25}$ E. Y. Tsymbal, A. Sokolov, I. F. Sabirianov, and B. Doudin, Phys. Rev. Lett. 90, 186602 (2003).

${ }^{26}$ H. Yang, S.-H. Yang, D.-C. Qi, A. Rusydi, H. Kawai, M. Saeys, T. Leo, D. J. Smith, and S. S. P. Parkin, Phys. Rev. Lett. 106, 167201 (2011).

${ }^{27}$ C. Kaiser, A. F. Panchula, and S. S. P. Parkin, Phys. Rev. Lett. 95, 047202 (2005). 